\title{
On the numerical simulation and convergence study for system of non-linear fractional dynamical model of marriage
}

\author{
M. Mohamed Khader ${ }^{1}$, Aml Shloof ${ }^{2}$ and Halima Ali ${ }^{3}$ \\ ${ }^{1}$ Department of Mathematics, Faculty of Science, Benha University, Benha, Egypt \\ ${ }^{2}$ Department of Mathematics, Faculty of Science, Al-Jabal Al-Gharbi University, Al-Zintan, Libya \\ ${ }^{3}$ Department of Mathematics, Faculty of Education, Aljufra University, Waddan, Aljufra, Libya
}

Received: 23 April 2017, Accepted: 14 June 2017

Published online: 8 November 2017.

\begin{abstract}
In this article, an implementation of an efficient numerical method for solving the system of coupled non-linear fractional (Caputo sense) dynamical model of marriage (FDMM) is introduced. The proposed system describes the dynamics of love affair between couples. The method is based on the spectral collocation method using Legendre polynomials. The proposed method reduces FDMM to a system of algebraic equations, which solved using Newton iteration method. Special attention is given to study the convergence analysis and deduce an error upper bound of the resulting approximate solution. Numerical simulation is given to show the validity and the accuracy of the proposed method.
\end{abstract}

Keywords: Coupled non-linear fractional dynamical model of marriage, Caputo fractional derivatives, Legendre collocation method, Convergence analysis.

\section{Introduction}

Fractional differential equations (FDEs) have been the focus of many studies due to their frequent appearance in various applications in fluid mechanics, biology, physics and engineering [19]. Consequently, considerable attention has been given to the solutions of FDEs and integral equations of physical interest. In last decades, fractional calculus has drawn a wide attention from many physicists and mathematicians, because of its interdisciplinary application and physical meaning [17]. Fractional calculus deals with the generalization of differentiation and integration of non-integer order. Most FDEs do not have exact solutions, so approximate and numerical techniques ([3], [6], [8], [11], [12]) must be used. Several numerical methods to solve FDEs have been given such as, HPM [5], FDM ([7], [14], [23]) and collocation method ([13], [22]).

In recent decades the study of interpersonal relationships has begun to be popular. Interpersonal relationships appear in many contexts, such as in family (marriage), kinship, acquaintance, work and clubs [2]. Mathematical modeling in interpersonal relationships is very important for capturing the dynamics of people. But there are few models in this area and models have been restricted to integer order differential equations. Marriage has been studied scientifically for the past sixty years, and we can draw some general conclusions that guide our modeling of marital interaction [4]. Researchers are motivated by trying to understand why some couples divorce, but others do not, and why, among those who remain married, some are happy and some are miserable with one another. There are high levels of divorce in 
today's world. In the United States the current estimate is that within a forty year span approximately $50 \%$ to $67 \%$ percent of firs marriages will end in divorce; the figure is $10 \%$ higher for second marriage [15]. Although the United States has the highest raw divorce over time exist worldwide. Since experiments in these areas are difficult to design and may be constrained by ethical considerations, mathematical models can play a vital role in studying the dynamics of marriage and behavioral features.

The earliest known linear model of marriage is Romeo and Juliet model [21]. Suppose that at any time $t$, we could measure Romeo's love or hate for Juliet, $R(t)$, and Juliet's love or hate for Romeo, $J(t)$. Positive values of these functions indicate love, and negative values indicate hate. A very simple assumption would be that the change in Romeo's love for Juliet is a fraction of his current love plus a fraction of her current love. Similarly, Juliet's love for Romeo will change by a fraction of her current love for Romeo and a fraction of Romeo's love for her. This assumption leads us to the model equations

$$
\frac{d R}{d t}=a R(t)+b J(t), \quad \frac{d J}{d t}=c R(t)+d J(t)
$$

where $a, b, c$, and $d$ are constants.

Representation of a function in terms of a series expansion using orthogonal polynomials is a fundamental concept in approximation theory and the basis of the solution of differential equations [24]. Legendre polynomials are widely used in numerical computation. One of the advantages of using Legendre polynomials as a tool for expansion functions is the good representation of smooth functions by finite Legendre expansion provided that the function $u(t)$ is infinitely differentiable. In [10], Khader and Hendy introduced an efficient numerical method for solving the fractional delay differential equation using the shifted Legendre polynomials. In [14] the Legendre polynomials were used to compute a spectral solution of a non-linear boundary value problems. This technique reduces the problem to a system of non-linear algebraic equations [8].

The main aim of the presented paper is concerned with the application of the Legendre collocation method to introduce the numerical simulation of the system of coupled non-linear FDMM and study the convergence analysis of the proposed method and estimate an upper bound of the resulting error of the proposed approximate solution.

\section{Preliminaries and notations}

In this section, we present some necessary definitions and mathematical preliminaries of the fractional calculus theory that will be required in the present paper.

Definition 1.The Caputo fractional derivative operator $D^{\alpha}$ of order $\alpha$ is defined as follows

$$
D^{\alpha} f(t)= \begin{cases}\frac{1}{\Gamma(m-\alpha)} \int_{0}^{t} \frac{f^{(m)}(\xi)}{(t-\xi)^{\alpha-m+1}} d \xi, & 0 \leq m-1<\alpha<m, \\ f^{(m)}(t), & \alpha=m \in \mathbb{N} .\end{cases}
$$

Similar to integer-order differentiation, Caputo fractional derivative operator is linear

$$
D^{\alpha}\left(c_{1} p(t)+c_{2} q(t)\right)=c_{1} D^{\alpha} p(t)+c_{2} D^{\alpha} q(t)
$$


where $c_{1}$ and $c_{2}$ are constants. For the Caputo's derivative we have $D^{\alpha} C=0, \mathrm{C}$ is a constant [16].

$$
D^{\alpha} t^{n}= \begin{cases}0, & \text { for } n \in \mathbb{N}_{0} \text { and } n<\lceil\alpha\rceil \\ \frac{\Gamma(n+1)}{\Gamma(n+1-\alpha)} t^{n-\alpha}, & \text { for } n \in \mathbb{N}_{0} \text { and } n \geq\lceil\alpha\rceil\end{cases}
$$

We use the ceiling function $\lceil\alpha\rceil$ to denote the smallest integer greater than or equal to $\alpha$ and $\mathbb{N}_{0}=\{0,1, \ldots\}$. For more details on fractional derivatives definitions and its properties see [20].

\section{Mathematical model}

In this paper, we study the behavior of the approximate solution of the fractional order system for the dynamics of love affair between couples [18]. The system which describes this model is given in the following form

$$
\begin{aligned}
& D^{\alpha} u(t)=-\lambda_{1} u(t)+\mu_{1} v(t)\left(1-\varepsilon v^{2}(t)\right)+v_{1}, \\
& D^{\alpha} v(t)=-\lambda_{2} v(t)+\mu_{2} u(t)\left(1-\varepsilon u^{2}(t)\right)+v_{2}, \quad t \in(0, L),
\end{aligned}
$$

with the initial conditions

$$
u(0)=u^{0}, \quad v(0)=v^{0},
$$

where $0<\alpha \leq 1, \lambda_{i}>0, \lambda_{i}, \mu_{i}$ and $v_{i}(i=1,2)$ are real constants. These parameters are oblivion, reaction and attraction constants. In the above equations, we assume that feelings decay exponentially fast in the absence of partners. The parameters specify the romantic style of individuals 1 and 2 .

Recently, several authors, for example ([2], [4]) have investigated the nonlinear FDMM and its special properties. For more details on the proposed model see ([15], [18]).

\section{An approximate formula of the Caputo fractional derivative}

The well-known Legendre polynomials are defined on the interval $[-1,1]$ and can be determined with the aid of the following recurrence formula [1]

$$
L_{k+1}(z)=\frac{2 k+1}{k+1} z L_{k}(z)-\frac{k}{k+1} L_{k-1}(z), \quad k=1,2, \ldots,
$$

where $L_{0}(z)=1$ and $L_{1}(z)=z$. In order to use these polynomials on the interval $[0,1]$ we define the so called shifted Legendre polynomials by introducing the change of variable $z=2 t-1$. Let the shifted Legendre polynomials $L_{k}(2 t-1)$ be denoted by $L_{k}^{*}(t)$. Then $L_{k}^{*}(t)$ can be obtained as follows

$$
L_{k+1}^{*}(t)=\frac{(2 k+1)(2 t-1)}{(k+1)} L_{k}^{*}(t)-\frac{k}{k+1} L_{k-1}^{*}(t), \quad k=1,2, \ldots
$$

where $L_{0}^{*}(t)=1$ and $L_{1}^{*}(t)=2 t-1$. The analytic form of the shifted Legendre polynomials $L_{k}^{*}(t)$ of degree $k$ is given by

$$
L_{k}^{*}(t)=\sum_{i=0}^{k}(-1)^{k+i} \frac{(k+i) !}{(k-i) !(i !)^{2}} t^{i}
$$


Note that $L_{k}^{*}(0)=(-1)^{k}$ and $L_{k}^{*}(1)=1$. The function $u(t)$, which is a square integrable function in $[0,1]$, may be expressed in terms of shifted Legendre polynomials as

$$
u(t)=\sum_{i=0}^{\infty} c_{i} L_{i}^{*}(t)
$$

where the coefficients $c_{i}$ are given by

$$
c_{i}=(2 i+1) \int_{0}^{1} u(t) L_{i}^{*}(t) d t, \quad i=0,1, \ldots
$$

In practice, only the first $(m+1)$-terms of shifted Legendre polynomials are considered. Then we have

$$
u_{m}(t)=\sum_{i=0}^{m} c_{i} L_{i}^{*}(t)
$$

The main approximate formula of the fractional derivative is given in the following theorem.

Theorem 1. Let $u(t)$ be approximated by shifted Legendre polynomials as (7) and also suppose $\alpha>0$, then

$$
D^{\alpha}\left(u_{m}(t)\right)=\sum_{i=\lceil\alpha\rceil}^{m} \sum_{k=\lceil\alpha\rceil}^{i} c_{i} w_{i, k}^{(\alpha)} t^{k-\alpha}
$$

where $w_{i, k}^{(\alpha)}$ is given by $w_{i, k}^{(\alpha)}=\frac{(-1)^{(i+k)}(i+k) !}{(i-k) !(k) ! \Gamma(k-\alpha+1)}$.

Proof. Since the Caputo's fractional differentiation is a linear operation we have

$$
D^{\alpha}\left(u_{m}(t)\right)=\sum_{i=0}^{m} c_{i} D^{\alpha}\left(L_{i}^{*}(t)\right)
$$

Employing Eq.(1) in formula (4), it is clear that $D^{\alpha} L_{i}^{*}(t)=0, i=0,1, \ldots,\lceil\alpha\rceil-1, \alpha>0$. Therefore, for $i=\lceil\alpha\rceil,\lceil\alpha\rceil+$ $1, \ldots, m$, and by using Eq.(1) in (4) we get

$$
D^{\alpha} L_{i}^{*}(t)=\sum_{k=0}^{i} \frac{(-1)^{i+k}(i+k) !}{(i-k) !(k !)^{2}} D^{\alpha}\left(t^{k}\right)=\sum_{k=\lceil\alpha\rceil}^{i} \frac{(-1)^{i+k}(i+k) !}{(i-k) !(k !) \Gamma(k-\alpha+1)} t^{k-\alpha} .
$$

Eq.(10) leads to the desired result (8).

Theorem 2. [9] The Caputo fractional derivative of order $\alpha$ for the shifted Legendre polynomials can be expressed in terms of the shifted Legendre polynomials themselves in the following form

$$
D^{\alpha}\left(L_{i}^{*}(t)\right)=\sum_{k=\lceil\alpha\rceil}^{i} \sum_{j=0}^{k-\lceil\alpha\rceil} \Theta_{i, j, k} L_{j}^{*}(t), \quad i=\lceil\alpha\rceil,\lceil\alpha\rceil+1, \ldots,
$$

where

$$
\Theta_{i, j, k}=\frac{(-1)^{i+k}(i+k) !(2 j+1)}{(i-k) !(k) ! \Gamma(k-\alpha+1)} \times \sum_{r=0}^{j} \frac{(-1)^{j+r}(j+r) !}{(j-r) !(r !)^{2}(k-\alpha+r+1)}
$$




\section{Convergence analysis}

In this section, special attention is given to study the convergence analysis and evaluate an upper bound of the error for the proposed approximate formula and the approximate solution.

Lemma 1. [9] For any continuous function $u(t)$ defined on $[0,1]$ with bounded second derivative (i.e., $\left|u^{\prime \prime}(t)\right| \leq \theta$ for some constant $\theta$ ), then the coefficients of the shifted Legendre expansion (5) is bounded in the following form

$$
\left|c_{i}\right| \leq \frac{\sqrt{6} \theta}{(2 i-1) \sqrt{2 i-3}} .
$$

Theorem 3. [9] For any function $u(t)$ and under the two assumptions:

$1 . u(t)$ is continuous function on $[0,1]$;

2.u(t) has bounded second derivative (i.e., $\left|u^{\prime \prime}(t)\right| \leq \theta$ for some constant $\theta$ ).

Then its shifted Legendre approximation $u_{m}(t)$ defined in (7) converges uniformly to the exact solution $u(t)$. Moreover, we have the following accuracy estimation

$$
\left\|u(t)-u_{m}(t)\right\|_{L^{2}[0,1]} \leq \sqrt{6} \theta\left(\sum_{i=m+1}^{\infty} \frac{1}{(2 i-3)^{4}}\right)^{0.5} .
$$

Theorem 4. The error $\left|E_{T}(m)\right|=\left|D^{\alpha} u(t)-D^{\alpha} u_{m}(t)\right|$ in approximating $D^{\alpha} u(t)$ by $D^{\alpha} u_{m}(t)$ is bounded by [9]

$$
\left|E_{T}(m)\right| \leq\left|\sum_{i=m+1}^{\infty} c_{i}\left(\sum_{k=\lceil\alpha\rceil}^{i} \sum_{j=0}^{k-\lceil\alpha\rceil} \Theta_{i, j, k}\right)\right| .
$$

\section{Implementation of Legendre spectral method for solving system of FDMM}

In this section, we will implement the Legendre spectral collocation method to solve numerically the system of coupled non-linear fractional dynamical model of marriage. Test example is presented to validate the solution's scheme.

We consider the proposed system (2) with different values of the constants $\lambda_{1}, \lambda_{2}, \mu_{1}, \mu_{2}, \varepsilon, v_{1}, v_{2}$ with the initial conditions (3). In order to use the Legendre collocation method, we approximate $u(t)$ and $v(t)$ with $m=3$ as

$$
u(t) \simeq \sum_{i=0}^{3} u_{i} L_{i}^{*}(t), \quad v(t) \simeq \sum_{i=0}^{3} v_{i} L_{i}^{*}(t)
$$

Substitution from Eqs.(16) and Theorem 1 in (2) we obtain

$$
\begin{aligned}
& \sum_{i=\lceil\alpha\rceil}^{3} \sum_{k=\lceil\alpha\rceil}^{i} u_{i} w_{i, k}^{(\alpha)} t^{k-\alpha}=-\lambda_{1} \sum_{i=0}^{3} u_{i} L_{i}^{*}(t)+\mu_{1} \sum_{i=0}^{3} v_{i} L_{i}^{*}(t)\left(1-\varepsilon\left(\sum_{i=0}^{3} v_{i} L_{i}^{*}(t)\right)^{2}\right)+v_{1}, \\
& \sum_{i=\lceil\alpha\rceil k=\lceil\alpha\rceil}^{3} \sum_{i}^{i} v_{i, k}^{(\alpha)} t^{k-\alpha}=-\lambda_{2} \sum_{i=0}^{3} v_{i} L_{i}^{*}(t)+\mu_{2} \sum_{i=0}^{3} u_{i} L_{i}^{*}(t)\left(1-\varepsilon\left(\sum_{i=0}^{3} u_{i} L_{i}^{*}(t)\right)^{2}\right)+v_{2} .
\end{aligned}
$$


We now collocate Eqs.(17)-(18) at $(m+1-\lceil\alpha\rceil)$ points $t_{p}, p=0,1, \ldots, m-\lceil\alpha\rceil$ as

$$
\begin{aligned}
& \sum_{i=\lceil\alpha\rceil k=\lceil\alpha\rceil}^{3} \sum_{i}^{i} u_{i} w_{i, k}^{(\alpha)} t_{p}^{k-\alpha}=-\lambda_{1} \sum_{i=0}^{3} u_{i} L_{i}^{*}\left(t_{p}\right)+\mu_{1} \sum_{i=0}^{3} v_{i} L_{i}^{*}\left(t_{p}\right)\left(1-\varepsilon\left(\sum_{i=0}^{3} v_{i} L_{i}^{*}\left(t_{p}\right)\right)^{2}\right)+v_{1}, \\
& \sum_{i=\lceil\alpha\rceil k=\lceil\alpha\rceil}^{3} \sum_{i}^{i} v_{i} w_{i, k}^{(\alpha)} t_{p}^{k-\alpha}=-\lambda_{2} \sum_{i=0}^{3} v_{i} L_{i}^{*}\left(t_{p}\right)+\mu_{2} \sum_{i=0}^{3} u_{i} L_{i}^{*}\left(t_{p}\right)\left(1-\varepsilon\left(\sum_{i=0}^{3} u_{i} L_{i}^{*}\left(t_{p}\right)\right)^{2}\right)+v_{2} .
\end{aligned}
$$

For suitable collocation points we use roots of shifted Legendre polynomial $L_{m+1-\lceil\alpha\rceil}^{*}(t)$. In this case, the roots $t_{p}$ of shifted Legendre polynomial $L_{3}^{*}(t)$ in the interval $[0,600]$ are

$$
t_{0}=67.621, \quad t_{1}=300, \quad t_{2}=532.379 .
$$

Also, by substituting from Eq.(16) in the initial conditions (3) we can find

$$
\sum_{i=0}^{3}(-1)^{i} u_{i}=u^{0}, \quad \sum_{i=0}^{3}(-1)^{i} v_{i}=v^{0}
$$

Equations (19)-(20), together with the equations of the initial conditions (21), give $(2 m+2)$ of non-linear algebraic equations which can be solved using the Newton iteration method, for $u_{i}, v_{i}$ for $i=0,1, \ldots, m$.

\section{Numerical simulation}

In this section, we implement the proposed method to solve the system of coupled non-linear fractional dynamical model of marriage (2) in the interval $[0,600]$ with different values of the constants $\lambda_{1}, \lambda_{2}, \mu_{1}, \mu_{2}, \varepsilon, v_{1}$ and $v_{2}$, different values of the fractional derivative $\alpha$ and different initial values.

The obtained approximate solutions by means of the proposed method are shown in figures 1-9. Where in figures 1-3, we presented the behavior of the approximate solution with different values of initial values $\left(u^{0}, v^{0}\right)=(2,4)$, $\left(u^{0}, v^{0}\right)=(1,2)$ and $\left(u^{0}, v^{0}\right)=(0.5,1)$, respectively, and $m=5$ with final time $t=600$.

Also, in figures 4-9, we presented the behavior of the approximate solution at $\alpha=0.9$ with different values of other constants. From these figures, we can conclude that the obtained solution $u(t), v(t)$ confirms the natural behavior of the proposed system.

\section{Conclusion and remarks}

In this article, the Legendre spectral collocation method is implemented for solving the system of coupled non-linear fractional dynamical model of marriage. The properties of the Legendre polynomials are used to reduce the proposed model to the solution of a system of nonlinear algebraic equation which is solved by using Newton iteration method. Special attention is given to study the convergence analysis and to estimate an upper bound of the error of the derived formula and the approximate solution. From the behavior of the obtained numerical solutions using the suggested method we can see that the natural behavior of the proposed system is confirmed. So, we can show that this approach can be solved the problem effectively. It is evident that the overall errors can be made smaller by adding new terms in the series (16). All computations in this paper are done using Matlab 8. 

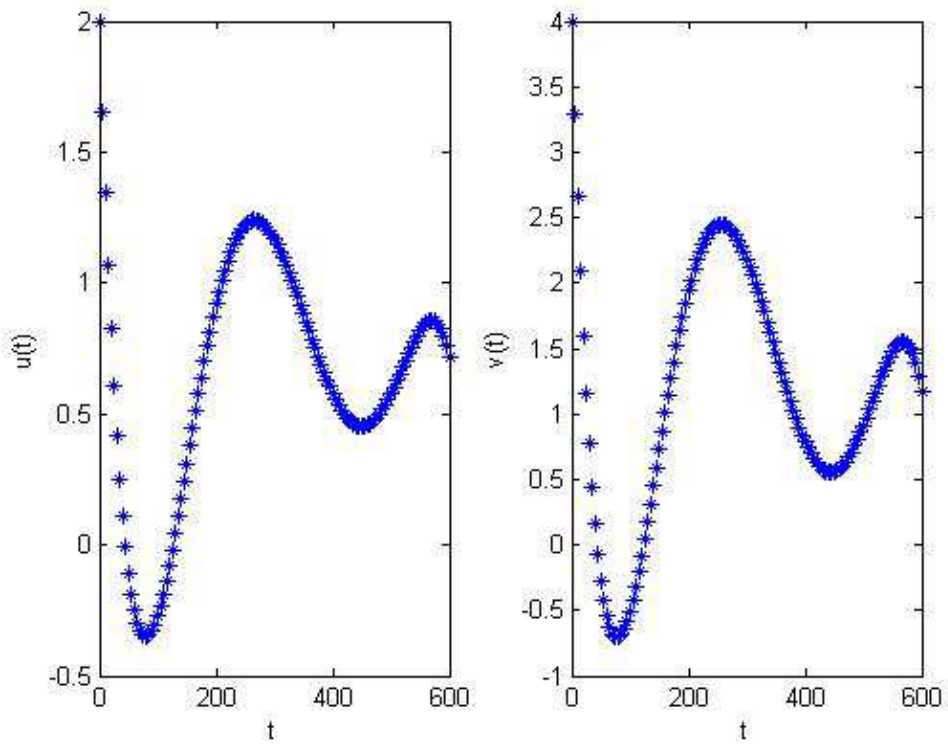

Fig. 1: The behavior of the approximate solution with $\alpha=0.6, u^{0}=2, v^{0}=4$ and $\lambda_{1}=0.001, \lambda_{2}=0.004, \mu_{1}=$ $0.005, \mu_{2}=-0.001, \varepsilon=0.01, v_{1}=0.02, v_{2}=0.03$.
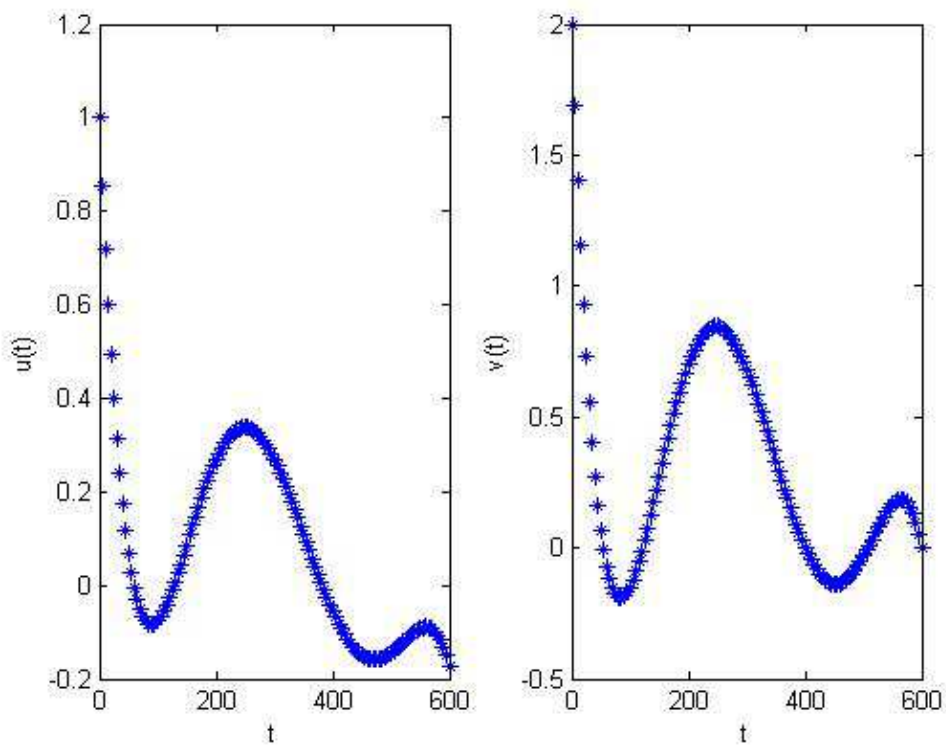

Fig. 2: The behavior of the approximate solution with $\alpha=0.6, u^{0}=1, v^{0}=2$ and $\lambda_{1}=0.001, \lambda_{2}=0.004, \mu_{1}=$ $0.005, \mu_{2}=-0.001, \varepsilon=0.01, v_{1}=0.02, v_{2}=0.03$. 

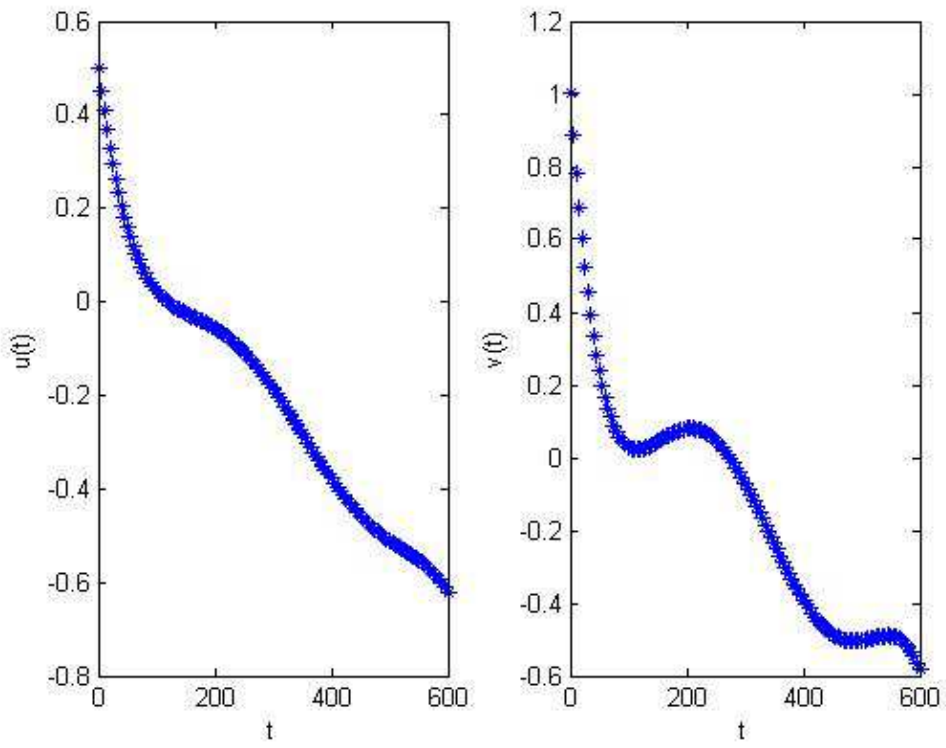

Fig. 3: The behavior of the approximate solution with $\alpha=0.6, u^{0}=0.5, v^{0}=1$ and $\lambda_{1}=0.001, \lambda_{2}=0.004, \mu_{1}=$ $0.005, \mu_{2}=-0.001, \varepsilon=0.01, v_{1}=0.02, v_{2}=0.03$.
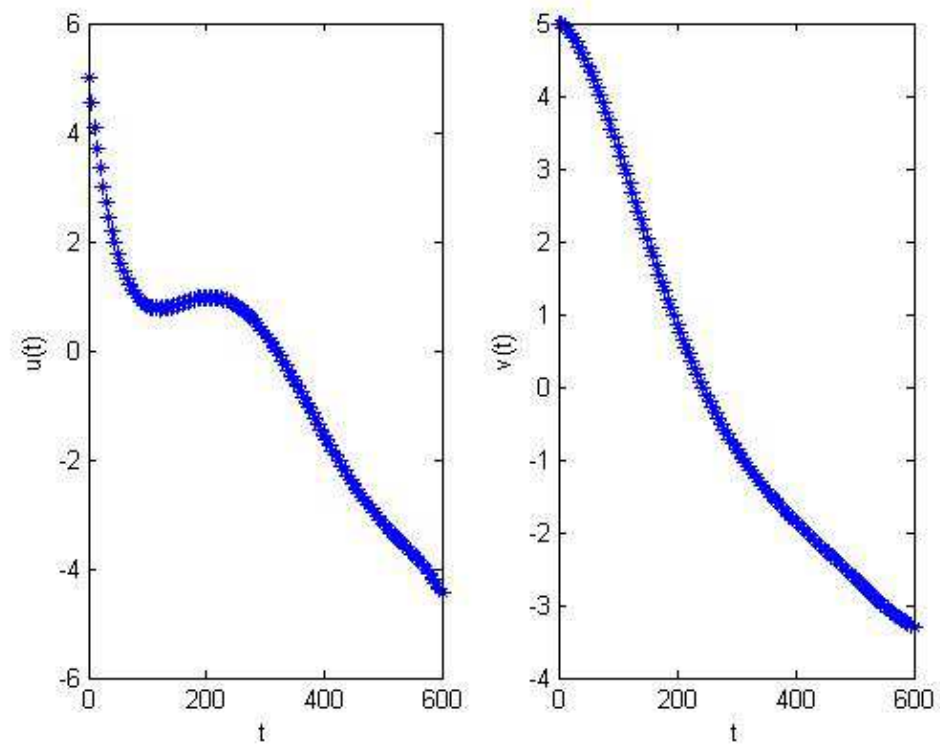

Fig. 4: The behavior of the approximate solution with $\alpha=0.9, u^{0}=v^{0}=5$ and $\lambda_{1}=0.001, \lambda_{2}=0.004, \mu_{1}=0.005, \mu_{2}=$ $-0.001, \varepsilon=0.01, v_{1}=0.02, v_{2}=0.03$. 

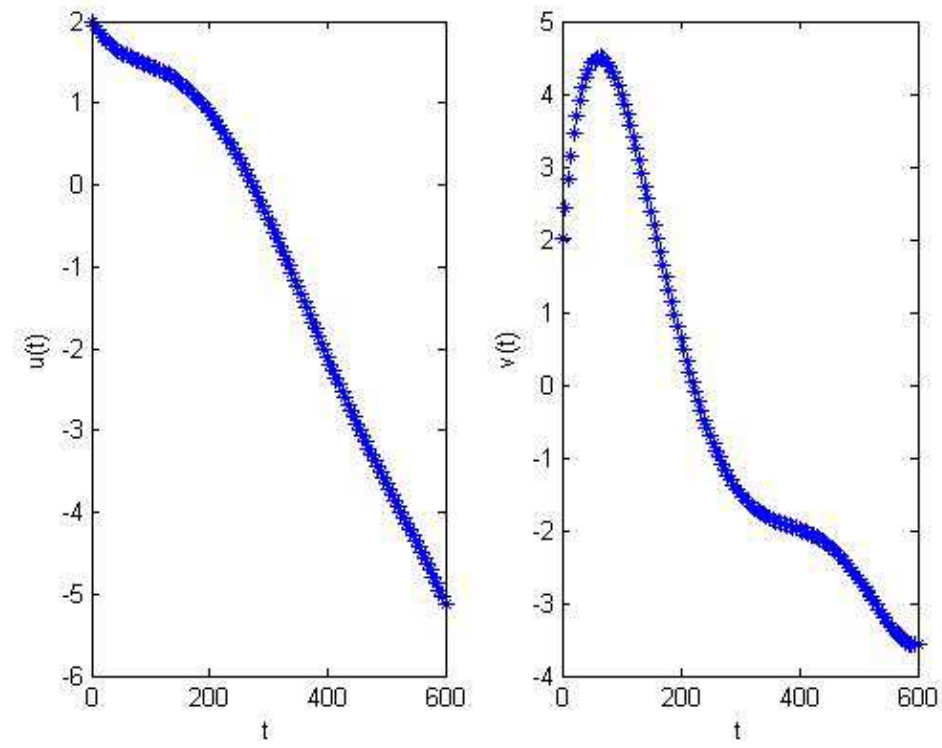

Fig. 5: The behavior of the approximate solution with $\alpha=0.9, u^{0}=v^{0}=2$ and $\lambda_{1}=0.001, \lambda_{2}=0.004, \mu_{1}=0.005, \mu_{2}=$ $-0.001, \varepsilon=0.01, v_{1}=0.02, v_{2}=0.03$.
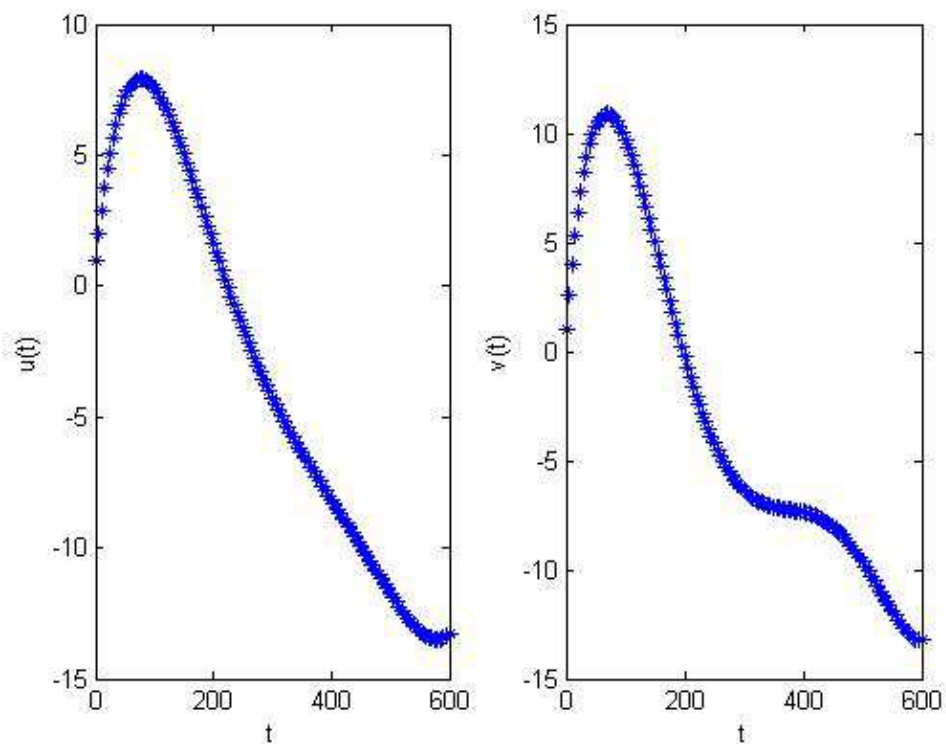

Fig. 6: The behavior of the approximate solution with $\alpha=0.9, u^{0}=v^{0}=0$ and $\lambda_{1}=0.001, \lambda_{2}=0.004, \mu_{1}=0.005, \mu_{2}=$ $-0.001, \varepsilon=0.01, v_{1}=0.07, v_{2}=0.09$. 

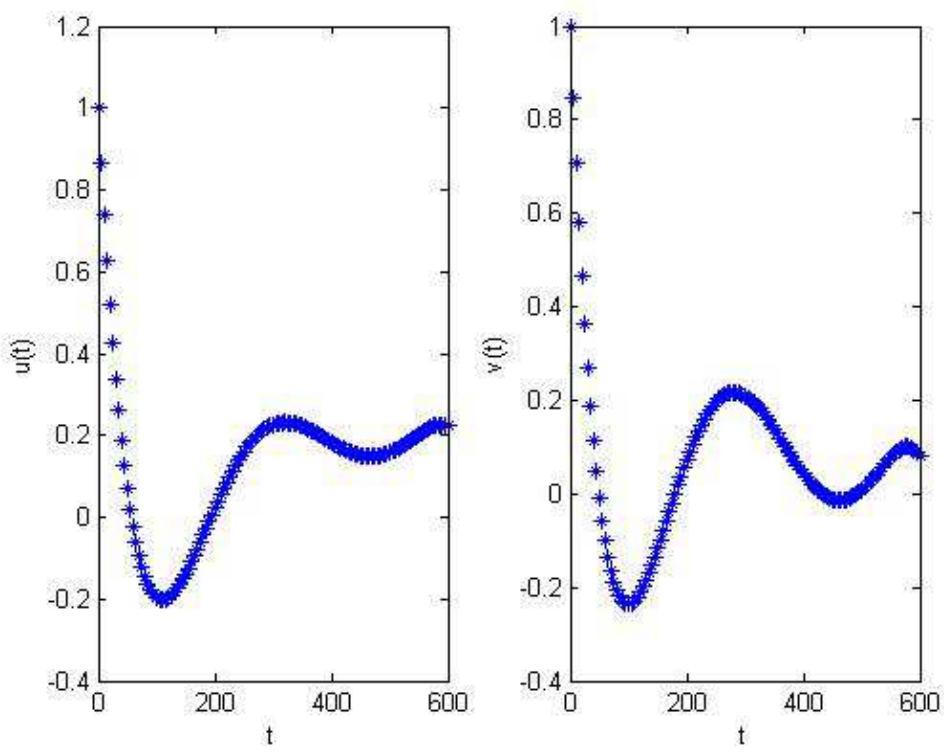

Fig. 7: The behavior of the approximate solution with $\alpha=0.9, u^{0}=v^{0}=1$ and $\lambda_{1}=0.001, \lambda_{2}=0.004, \mu_{1}=0.005, \mu_{2}=$ $-0.001, \varepsilon=0.01, v_{1}=0.0, v_{2}=0.0$.
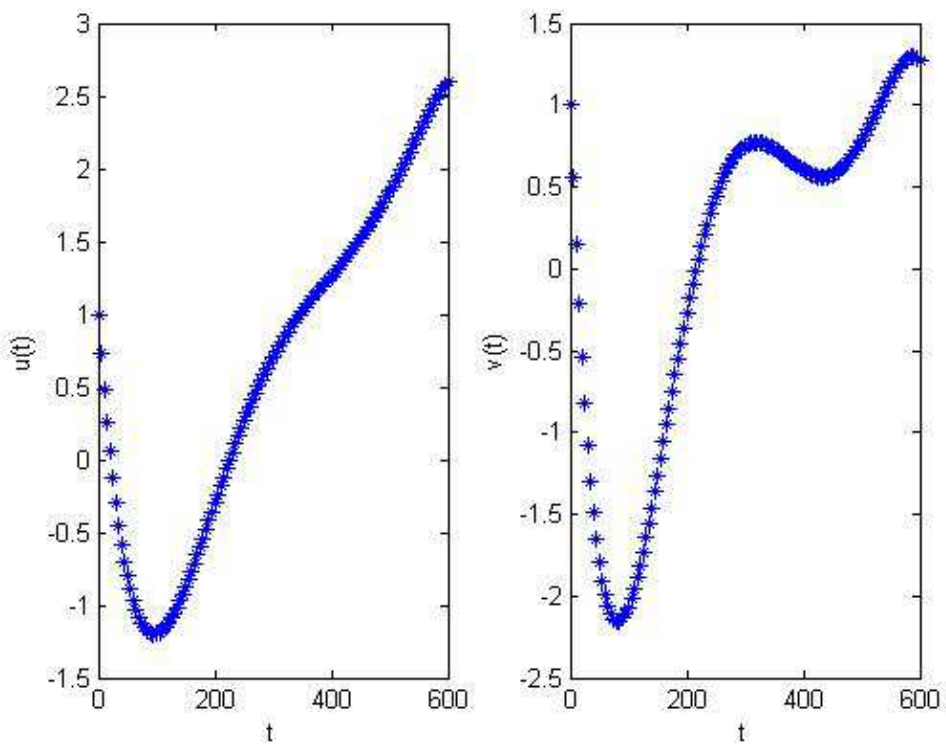

Fig. 8: The behavior of the approximate solution with $\alpha=0.9, u^{0}=v^{0}=1$ and $\lambda_{1}=0.001, \lambda_{2}=0.004, \mu_{1}=0.005, \mu_{2}=$ $-0.001, \varepsilon=0.09, v_{1}=-0.01, v_{2}=-0.01$. 

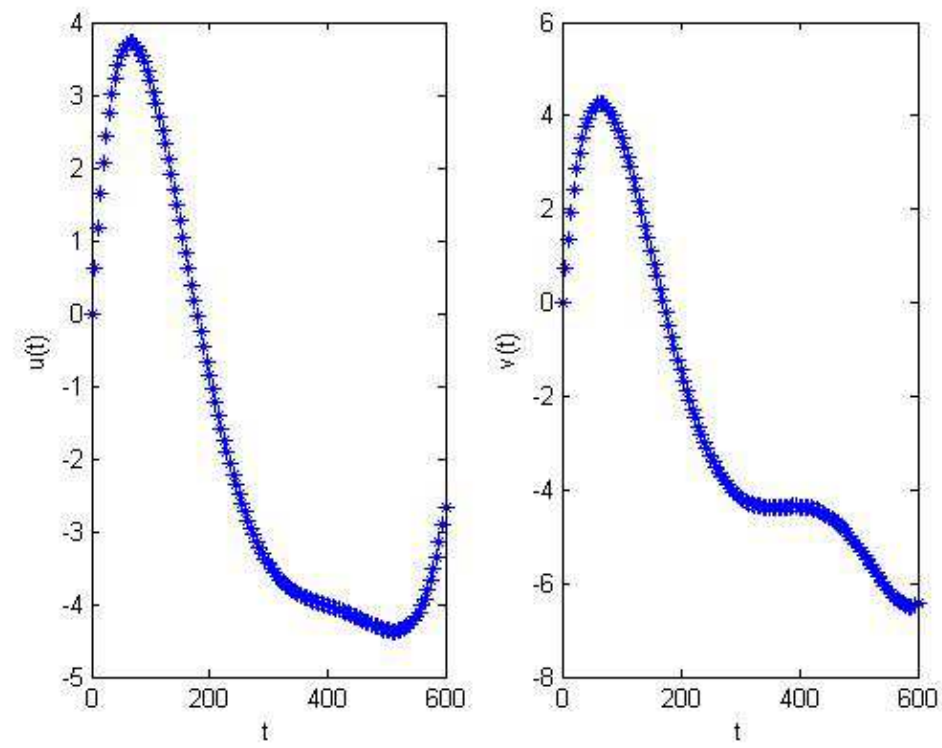

Fig. 9: The behavior of the approximate solution with $\alpha=0.9, u^{0}=v^{0}=0$ and $\lambda_{1}=0.003, \lambda_{2}=0.006, \mu_{1}=0.005, \mu_{2}=$ $-0.001, \varepsilon=0.01, v_{1}=0.05, v_{2}=0.05$.

\section{Competing interests}

The authors declare that they have no competing interests.

\section{Authors' contributions}

All authors have contributed to all parts of the article. All authors read and approved the final manuscript.

\section{References}

[1] Bell, W. W., Special Functions for Scientists and Engineers, Great Britain, Butler and Tanner Ltd, Frome and London, (1968).

[2] Cherif, A., and Barley, K., Stochastic nonlinear dynamics of interpersonal and romantic relationships, Applied Mathematics and Computation, 217, 6273-6281, (2011).

[3] Eid, A., and Khader, M. M., Numerical studies using FDM for viscous dissipation and thermal radiation efects on the slip flow and heat transfer due to a stretching sheet embedded in a porous medium with variable thickness and variable thermal conductivity, New Trends in Mathematical Sciences, 4(1), 38-50, (2016).

[4] Gottman, J. M., Murray, J. D, Swanson, C. C. Tyson, R., and Swanson, K. R., The Mathematics of Marriage, Cambridge, MA: MIT Press, (2002).

[5] Jafari, H. and Momani, S., Solving fractional diffusion and wave equations by modified homotopy perturbation method, Physics Letter A, 370, 388-396, (2007).

[6] Khader, M. M., On the numerical solutions for the fractional diffusion equation, Communications in Nonlinear Science and Numerical Simulation, 16, 2535-2542, (2011).

[7] Khader, M. M., Numerical treatment for solving the perturbed fractional PDEs using hybrid techniques, Journal of Computational Physics, 250, 565-573, (2013). 
[8] Khader, M. M., Numerical treatment for solving fractional Riccati differential equation, Journal of the Egyptian Mathematical Society, 21, 32-37, (2013).

[9] Khader, M. M., On the numerical solution and convergence study for system of non-linear fractional diffusion equations, Canadian Journal of Physics, 92(12), 1658-1666, (2014).

[10] Khader, M. M., and Hendy, A. S., The approximate and exact solutions of the fractional-order delay differential equations using Legendre pseudospectral method, International Journal of Pure and Applied Mathematics, 74(3), 287-297, (2012).

[11] Khader, M. M., and Sweilam, N. H., Numerical and analytical study for integro-differential equations using spectral collocation method, New Trends in Mathematical, 3(4), 144-153, (2015).

[12] Khader, M. M., and Hendy, A. S., A numerical technique for solving fractional variational problems, Mathematical Methods in Applied Sciences, 36(10), 1281-1289, (2013).

[13] Khader, M. M., EL-Danaf, T. S., and Hendy, A. S., A computational matrix method for solving systems of high order fractional differential equations, Applied Mathematical Modelling, 37, 4035-4050, (2013).

[14] Khader, M. M., Sweilam, N. H., and Mahdy, A. M. S., Numerical study for the fractional differential equations generated by optimization problem using Chebyshev collocation method and FDM, Applied Mathematics and Information Science, 7(5), 20112018, (2013).

[15] Martin, M. T. C., and Bumpass, B. L., Recent trends in marital disruption, Demography, 26(1), 37-51, (1989).

[16] Miller, K. S., and Ross, B., An Introduction to the Fractional Calculus and Fractional Differential Equations, John Wiley and Sons, New York, NY, USA, (1993).

[17] Oldham, K. B., and Spanier, J., The Fractional Calculus, Academic Press, New York, (1974).

[18] Ozalp, N., and Koca, I., A fractional order nonlinear dynamical model of interpersonal relationships, Advances in Difference Equations, 189(1), 1-7, (2012).

[19] Podlubny, I., Fractional Differential Equations, Academic Press, New York, (1999).

[20] Srivastava, H. M., Kilbas, A. A., and Trujillo, J. J., Theory and Application of Fractional Differential Equations, Elsevier, Amsterdam, (2006).

[21] Strogatz, S. H., Nonlinear Dynamics and Caos: With Applications in to Physics, Biology, Chemistry and Engineering, AddisonWesley, Reading, MA, (1994).

[22] Sweilam, N. H., and Khader, M. M., A Chebyshev pseudo-spectral method for solving fractional integro-differential equations, ANZIAM, 51, 464-475, (2010).

[23] Sweilam, N. H., Khader, M. M. and Nagy, A. M., Numerical solution of two-sided space-fractional wave equation using finite difference method, Journal of Computional and Applied Mathematics, 235, 2832-2841, (2011).

[24] Sweilam, N. H., Khader, M. M., and Mahdy, A. M. S., Numerical studies for fractional-order Logistic differential equation with two different delays, Journal of Applied Mathematics, 2012, Article ID 764894, 14 pages, (2012). 